\title{
Environmental exposure assessment using Indoor/Outdoor detection on smartphones
}

\begin{abstract}
We present an energy-efficient method for Indoor/Outdoor detection on smartphones. The creation of an accurate environmental exposure detection method enables crucial advances to a number of health sciences, which seek to model patients' environmental exposure. In a field trial, we collected data from multiple smartphone sensors, along with explicit indoor/outdoor labels entered by participants. Using this rich dataset, we evaluate multiple classification models, optimized for accuracy and low energy consumption. Using all sensors, we can achieve $99 \%$ classification accuracy. Using only a subset of energy-efficient sensors we achieve $92.91 \%$ accuracy. We systematically quantify how subsampling can be used as a trade-off for accuracy and energy consumption. Our work enables researchers to quantify environmental exposure using commodity smartphones.
\end{abstract}

Keywords: Energy efficiency, environmental exposure, indoor/outdoor detection, smartphones.

\section{INTRODUCTION}

We present an energy-efficient method for Indoor/Outdoor detection on smartphones. Developing an accurate detection method enables crucial advances to a number of health sciences which seek to model patients' environmental exposure [29]. In general, because the air quality varies substantially between indoor and outdoor settings [3], accurate longitudinal measurements from smartphones could provide valuable scientific evidence for a number of disciplines. For instance, epidemiologists are interested in quantifying environmental exposure during the pollen season to better understand its effect on allergies and asthma [16]. Quantifying the environmental exposure of pregnant women can lead to better understanding of the link between pollution and birth size [35], infant intelligence [36], cognitive development [6], and the advancement of puberty [18].

Many such studies rely on self-reported questionnaires to quantify environmental exposure, which may be unreliable and inconsistent as users may forget to report. Since people tend to carry their smartphone on a daily basis [13], an automated longitudinal monitoring technique on smartphones is affordable, easier to automate, and can greatly improve existing methodologies for quantifying environmental exposure.

Previous research on fingerprinting and localisation techniques can potentially be used for Indoor/Outdoor detection. For example, such techniques can be used to extrapolate environmental exposure, provided that an environmental mapping is available that characterises each particular location as indoor or outdoor. Similarly, activity recognition can be used to extrapolate environmental exposure. This would require a reliable mapping between the specific activity that is detected, and its associated environmental exposure. Location-based services can also be used, assuming a reliable mapping between a specific service that is launched and its associated environmental exposure.

However, using such techniques for indoor/outdoor detection is challenging because it requires instrumentation of the environment (e.g., by installing beacons or tags), or an a priori mapping of the environment. On the other hand, in our work we seek to provide an energy-efficient Indoor/Outdoor detection on commodity smartphones.

We present a study where we collected sensor data from several participants, and simultaneously collected ground-truth indoor/outdoor labels from an independent handset. We tackle indoor/outdoor detection of a mobile user as a classification problem, and we balance the trade-off between power consumption and prediction accuracy for quantifying environmental exposure. Previous work has noted that energy efficiency can be increased by relying on lightweight smartphone sensors and subsampling. However, it remains unclear what is the tradeoff with the accuracy of the system. Our work seeks to quantify environmental exposure using smartphones, and consider both prediction accuracy and energy efficiency.

The rest of the paper is organised as follows. Section II starts with an overview of previous approaches for localisation, activity recognition, location-based services and environmental exposure measurement techniques. Section III describes our experiment, our measurements and systematic approach to quantify environmental exposure from smartphones' sensor data. Section IV describes the data analysis and feature selection for our detection models and Section V provides an exhaustive evaluation of such models: lower energy consumption vs higher prediction accuracy vs features required. Section VI frames our work within literature and demonstrates potential applications of our model in practice. Section VII concludes our manuscript. 


\section{RELATED WORK}

While very little work has explicitly addressed the measurement of environmental exposure using smartphones there is a substantial literature on localisation techniques for mobile technologies, and how they can be used in indoor and outdoor settings [10]. While these techniques do not explicitly address environmental exposure, they can be used for such purposes provided that a mapping is available to characterise locations as indoor or outdoor.

\subsection{Localisation}

A variety of fingerprinting and localisation techniques have been reported in literature. These techniques can be used to extrapolate environmental exposure, provided that an environmental mapping is available to characterise each particular location as indoor or outdoor. For instance, localisation via tagging or fingerprinting can be used to infer a device's environment. One such approach is the use of Radio Frequency Identification (RFID) [31] or Bluetooth tags [33], whereby tags are placed in known positions in the environment and can be used as a frame of reference to infer the position of the user, and ultimately environmental exposure. A participatory sensing approach can also be used to train the classification model [47], thus alleviating bootstrapping issues in such methods. Quite often, indoor localisation uses Received Signal Strength (RSS) as a metric for location fingerprinting [15,20,46,50]. RSS fingerprints are then mapped to relative spatial coordinates which are geographically connected to user movement paths. For example, Borealis used WiFi RSS for outdoor localization via fingerprinting [52], and systems such as LocataNet show how bootstraping issues can be addressed by autonomously propagating the positioning network in a variety of environments [7].

While many localisation techniques are implemented as a replacement for GPS, often GPS is still used in localisation. For example, a wireless mobile indoor/outdoor tracking system was designed to track the absolute position of all nodes in a network [30]. The system uses GPS when the signal is available, and Radio Frequency (RF) otherwise. Similarly, uLocate performs indoor location tracking by incorporating a WiFi and GPS sensing [11]. The system is efficient in monitoring daily movements and location behavior of elder disabled people who have serious health issues.

In environments where $\mathrm{WiFi}$ is sparse, research has shown that incorporating user movement activity from accelerometer, magnetometer and gyroscope smartphone sensors, along with WiFi fingerprinting, can provide robust localisation [48]. This approach uses Bayesian filters to detect movement and estimate direction changes. Additionally, localisation can be performed in instrumented environments. For example, the use of non-invasive audio has been proposed for localisation [25]. Such an acoustic indoor positioning system can be compatible with smartphones and requires synchronized acoustic beacons to be installed in the environment.

Systems that are used for indoor navigation can also be used to extrapolate environmental exposure. For example, real time indoor navigation on smartphones is feasible by using accelerometer, gyroscope, magnetometer and WiFi sensor data [9]. This approach uses WiFi fingerprinting, geomagnetic fingerprinting and map matching for indoor navigation. In such settings, it is also possible to incorporate the use of a barometer [24], to provide accurate elevation measurement without special infrastructure.

\subsection{Activity recognition}

A number of projects have implemented activity recognition, which can be used to extrapolate environmental exposure. This assumes that there can be a reliable mapping between the specific activity that is detected, and its associated environmental exposure. For example, SurroundSense can perform activity recognition using fingerprinting of ambient sound, light and color measured using the microphone and camera [5]. In this case, the system uses a Support Vector Machine (SVM) classifier to infer the activity of the user. The Jigsaw sensing engine uses continuous sensing for activity recognition on smartphones [27]. It uses accelerometer, microphone and periodic GPS sensing to infer user activity by incorporating a J48 tree-based classifier.

Semi-Supervised learning has been used to detect activity from cell signal, light and magnetic field sensors [38]. This approach uses a semi-supervised co-training model based on Naive Bayes classifier which proved to be efficient on both accuracy and energy consumption. In this case, the authors explicitly avoid the use of GPS due to its highenergy cost. A similarly energy-aware approach is the SenseLess system [8], which is able to dynamically choose the most energy efficient smartphone sensors for activity recognition. 


\subsection{Location-Based Services}

A substantial literature on Location-Based Services exists, which can also be used to extrapolate environmental exposure. This assumes that there can be a reliable mapping between a specific service that is launched, and its associated environmental exposure. For instance, Indoor Location Based Services (LBS) have been proposed with the use of ultrasonic signal acquisition [23]. Such a system is based on a microcontroller which exploits context transmitted by ultrasonic beacons placed on the ceilings of the buildings, and Bluetooth smartphone sensor for indoor tracking. Similarly, smartphone logs have been used for location recognition and prediction [12]. The system uses machine learning techniques in order to infer the user movement activity by incorporating k-Nearest Neighbors and Decision Trees classifiers as well as Hidden Markov Models. The models are applied on data collected from GPS and WiFi sensing.

\subsection{Environmental exposure on smartphones}

The literature on localisation, activity recognition, and location-based services does not explicitly attempt to quantify environmental exposure on smartphones. As a result, these methods are not ideal because they either require instrumentation of the environment (e.g., by installing beacons or tags), or an a priori mapping of the environment which may not always be available.

However, some projects have attempted to infer environmental exposure, and react to the user being present in an indoor or outdoor setting. For example, Ouchi \& Doi [34] report on a system that incorporates an indoor and outdoor activity recognition engine. This system switches between indoor or outdoor engine based on the availability of GPS signal, and therefore makes that assumption that when GPS is available, the user is outdoors. The paper does not provide a detailed assessment on the accuracy of this assumption. Similarly, a lifelog system correlated user position, activity, and experience to detect and predict user behaviour [28]. This system switches between indoor activity detection and outdoor activity detection based on the availability of GPS signal or Bluetooth beacons, and thus requires instrumentation of the environment.

Some projects have explicitly quantified environmental exposure on smartphones. For instance, IODetector is an Android application that recognises the current environment state by incorporating light sensor, magnetometer, and cell tower signal strength [21]. The prediction accuracy of this model is $85 \%$, and is relatively energy efficient as GPS is discarded. Researchers have also shown how GPS can be used to infer environmental exposure when coupled with a magnetometer [32] or a light sensor [49] with prediction accuracy of $96.5 \%$ and $90 \%$, respectively.

An important shortcoming of work that has explicitly considered environmental exposure is the limited attention paid to energy efficiency. Energy gains can be achieved by relying on lightweight smartphone sensors [51], however it is not clear what is the trade-off with the accuracy of the system. Similarly, it is possible to reduce the duty cycle of sensors to save energy [51], however it is not clear what the impact will be on the accuracy of the system.

Furthermore, an important limitation is that ground-truth (i.e., whether a user is actually indoors or outdoors) has been typically collected through user labelling on the same phone that does the environmental sensing during the experiment. This can be problematic since user labelling may affect the collected sensor data, and therefore introduce bias in the analysis.

To summarise, literature on activity recognition in indoor settings typically uses lightweight sensors and avoids GPS. Activity recognition research that operates in both indoor and outdoor settings uses GPS to detect indoor vs. outdoor settings, and results in high energy consumption. Additionally, some methods use subsampling to save power. In our work, we aim for high accuracy and high energy efficiency in indoor/outdoor detection, by identifying a subset of sensors that optimise those requirements. In addition, we explicitly consider the impact of subsampling on energy consumption and accuracy.

\section{EXPERIMENT}

\subsection{Data collection}

We collect data from multiple smartphone sensors, and simultaneously collect ground-truth user-provided label data with another smartphone. The sensors we consider are:

- Activity context is provided by Google's Activity Recognition API and provides the user's physical activity context. Logged every 5 minutes. 
- Barometric pressure is logged at $5 \mathrm{~Hz}$. Barometric pressure data is collected since buildings may have a controlled environment, which regulates the pressure inside the building to be either positive or negative, depending on the yearly season and the height of the building. This context can be used to infer the indoor/outdoor setting of the user.

- The amount of ambient light is collected through the light sensor. The sun provides up to about 100,000 lux [39], indoor lighting provides between 300 and 750 lux [19,22], and the moon between $0.27-1.0$ lux under a clear sky [39].

- The phone may be in a pocket, thus obstructing the light sensor. This is addressed by collecting data from the proximity sensor, which is often physically adjacent to light sensor. Data was collected every time a change occurs (i.e., a binary value which denotes "close" or "distant").

- Clouds can significantly affect the reading. This can be addressed by using publicly available APIs that provide cloud coverage data ( 0 being no clouds and 100 being completely clouded), given the user's location, captured every 5 minutes.

- Time of day can significantly affect the amount of ambient light. We divide the day into certain parts of varying expected light: Day, Night and Twilight. Twilight was used both for sunrise and sunset since they have the same amount of light. The times of sunrise and sunset were calculated using the algorithm provided by the Almanac for Computers [1,40] given the date, time and geolocation of the phone.

- GSM signal strength is collected for both the active tower and neighbouring towers, every time the phone connects to a new GSM tower. When moving from outdoors to indoors (or visa versa), the GSM signal strength abruptly changes due to building walls [21].

- Accelerometer data is collected at $5 \mathrm{~Hz}$. We include accelerometer data to account for the stochastic dependences of the acceleration with the other data. Our hypothesis is that having minimal or maximal values of acceleration can help detect if the user is either indoor or outdoor.

- Magnetometer variance data is collected at $5 \mathrm{~Hz}$. Magnetometers are sensitive to disturbances caused by electronics, magnets and metals [26]. Our hypothesis is that more magnetic disturbance is detected indoors than when outdoors.

- Ambient noise levels are recorded using the microphone, specifically both decibel levels and frequency. Sound acoustics are different between indoors and outdoors environments. The software listens for 30 seconds, every five minutes and is processed with AWARE's Ambient Noise Plugin [4].

- The number of active GPS satellites is recorded. GPS needs to have a direct line of sight between the satellite and the phone's antenna. For indoors, the number of visible satellites is lower than outdoors. We log one GPS entry every five minutes. We wait for a first fix up to 40 seconds. This was intended to give the phone a chance to get a fix on the satellites, but also avoid spending too much time to find satellites when they are unreachable (i.e., when indoors). If the location of the user is determined by the GPS, then we store and used this last known location when logging cloud coverage and the day's period.

- Screen status data is recorded by listening to event broadcasts by Android. Our hypothesis is that there is a difference in phone usage between indoor and outdoor settings $[17,37,45]$.

- The number of visible WiFi APs is recorded every 5 minutes. Our hypothesis is that there is a difference on the amount of visible WiFi AP when indoors and outdoors.

\subsection{Experiment Design}

Our participants used their personal Android smartphones as the primary device for data collection. In addition, we gave participants a "labelling" device: a secondary smartphone to record when they entered indoors or outdoors. The labelling data was transmitted via Bluetooth to the primary phone for storage. This was a necessary inconvenience to participants, as we minimise potential sensor sampling bias on the primary device, i.e., using the primary phone for labelling would entail taking it out of their pocket, shifting its orientation, and generally affecting many of the sensor readings.

The secondary phone ran a single labelling application (Fig. 1). It enabled users to indicate whether they have moved to an indoor or outdoor setting, and more precisely to indicate if this happened "Now," "1 min ago" or " 5 mins ago" to provide the labelling of transitions even if they had forgotten. Finally, the participants' primary phone showed a reminder during the experiment, remaining always visible (Fig. 2). 
The notification showed the most recent label that the participant had indicated ("indoor"/"outdoor") and also provided labelling options. During briefing, participants were instructed on how to deal with certain ambiguous settings. Specifically, we asked them to label buses and cars as "indoors," and balconies as "outdoors." In addition, we asked them to label all indoor/outdoor transitions they made, and to be consistent in how they label ambiguous settings.

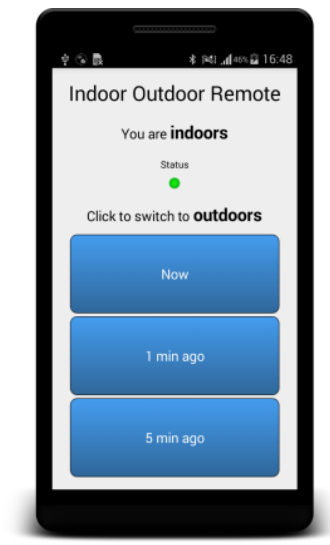

Fig. 1. Graphical user interface on the secondary phone.

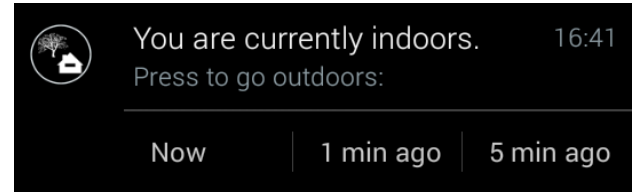

Fig. 2. Notification to label data.

Participants were recruited for a one-day deployment each, and asked to explore their urban space at home and work. This allowed us to maximise the variation within individual datasets, while also minimise the burden on each participant. During debriefing, we individually discussed with the participants the logged transitions, and recalled the places and activities associated with each transition.

In addition, we collected an "extreme use-case" dataset, used to test our prediction model. This independent dataset is intended to pose a challenge to our predictive model, and we expect our model to perform poorly in this dataset. Specifically, we collected data from a windowless basement, from indoors locations near big windows and glass walls, in an urban canyon (outdoor yard surrounded by very tall walls). We also collect data by repeatedly transitioning between an indoor and outdoor location, once every 30 seconds. All extreme cases were collected under two settings: with the smartphone either in a pocket or in hand. For each condition (e.g., indoor, outdoor), we collected data for a total of 30 minutes, 20 minutes for repeated transitions. Lastly, we collect data from an altogether different country and city.

\subsection{Data treatment and coding}

The collected data was treated and coded as follows:

- Activity data was coded as: 0 (in vehicle), 1 (bicycle), 2 (on foot), 3 (still), 4 (unknown), and 5 (tilting). This data is calculated through Google's Activity Recognition API which uses low power sensors [2].

- Barometric pressure data was not treated. The values of this feature range from 997 to $1009 \mathrm{mBar}$.

- Ambient luminance data was not treated. The values of this feature range from 0 to 10000 lux.

- Proximity data was coded as: 0 (obscured), and 1 (not obscured).

- Cloud coverage data was not treated. The values are continuous from 0 to $100 \%$. In cases where a recent location fix was not available, the value of this feature is exceptionally set to 0 .

- Time of day data was coded as: 1 (twilight), 2 (night), 3 (day) and -1 (unknown if no location fix is available).

- GSM signal strength data was not coded. The values of this feature range from 0 to 31 asu. Note that the relationship between asu and $\mathrm{dBm}$ is: $1 \mathrm{dBm}=-113+2 *$ asu, which means that 0 asu is $-113 \mathrm{dBm}$ or less, and 31 asu means $-51 \mathrm{dBm}$ or greater.

- GSM neighboring towers signal strength was treated as follows. We computed the average signal strength of all the towers that are visible to the smartphone. The values of this feature range between -121 and $10 \mathrm{dBm}$.

- Accelerometer data was not treated. The values of this feature range from 0.01 to $10.67 \mathrm{~m} / \mathrm{s}^{2}$. It does not include the force of gravity. 
- Magnetometer variance data was treated as follows. We calculate the variance in the magnetometer data on each axis $(x, y, z)$ using an 18-second sliding window [21]. In addition, we calculate the total average by summing variance of each axis. The values of this feature range from 1 to 9000 in $\mu \mathrm{T}^{2}$.

- Ambient noise data was not treated. The values of this feature range from 13 to 56 decibels.

- Noise frequency data was not treated. The values of this feature range from 8 to $310 \mathrm{~Hz}$.

- Visible GPS satellites data was not treated, and ranges from 0 to 13 satellites.

- Screen status data was coded as: 0 (off), 1 (on), 2 (locked), 3 (unlocked).

- WiFi AP data was not treated, and ranges from 0 to 22 APs.

- Indoor/outdoor labels were coded as follows: 0 (indoor), 1 (outdoor).

Due to the variety of sampling frequencies, we further processed all data to upsample or downsample it to $1 \mathrm{~Hz}$. Downsampling is achieved by averaging, while upsampling is achieved by replicating the last known value, both common data treatment techniques.

Our power consumption values are estimates provided by Qualcomm's Trepn Power Profiler [42]. We used an LG Nexus 5 that is a Snapdragon device and is fully supported by Trepn's hardware instrumentation. We used the same device in all our power consumption measurements to avoid any potential hardware variation bias. We first establish the baseline power consumption when idle. We then subtract this baseline from the estimated power consumption when each sensor is activated individually by our software, over a 20 -minute period as a baseline (Table I).

Table I

Characteristics of the extracted features

\begin{tabular}{llll} 
Feature & Power $(\mathbf{m W})$ & Range & Unit \\
Activity & 61.74 & $\{0, \ldots, 5\}$ & Net number \\
Barometric Pressure & 96.48 & {$[997,1009]$} & $\mathrm{mBar}$ \\
Ambient luminance & 15.30 & {$[0,10000]$} & lux \\
Proximity & 14.10 & $\{0,1\}$ & Net number \\
Cloud coverage & 67.39 & {$[0,100]$} & $\%$ \\
Time of day & 40.85 & $\{-1, \ldots 3\}$ & Net number \\
Gsm signal strength & 25.42 & {$[0,31]$} & asu \\
Gsm neighbouring towers signal strength & 25.42 & {$[-121,10]$} & $\mathrm{dBm}$ \\
Accelerometer & 86.31 & {$[0.01,10.67]$} & $\mathrm{m} / \mathrm{s}^{2}$ \\
Magnetometer variance & 77.49 & {$[1,9000]$} & $\mu \mathrm{T}^{2}$ \\
Ambient Noise & 40.37 & {$[13,56]$} & $\mathrm{decibel}$ \\
Noise frequency & 40.37 & {$[8,310]$} & $\mathrm{Hz}$ \\
Active satellites & 59.18 & $\{0, \ldots, 13\}$ & $\mathrm{Net}$ number \\
Screen status & 0.75 & $\{0, \ldots, 3\}$ & $\mathrm{Net}$ number \\
Number of WiFi APs & 33.64 & $\{0, \ldots, 22\}$ & $\mathrm{Net}$ number \\
\hline
\end{tabular}

\section{ANALYSIS}

We approach indoor/outdoor detection of a mobile user as a classification problem. To classify the environmental context of the user, we build a model which accepts as input the contextual features shown in Table I, and outputs a class attribute which is the Indoor/Outdoor feature. To assess the efficiency of our classification model we introduce two evaluation accuracy metrics. The first metric is the prediction accuracy $p$ defined as

$$
p=\frac{t p+t n}{t p+f p+t n+f n}
$$

where $t p$ is true positives, $t n$ is true negatives, $f p$ is false positives, and $f n$ is false negatives. In addition, a confidence interval is calculated for $p$, using a binomial test experiment. This is computed using as input the values $p$ 's numerator, denominator, and $p$ itself. The second metric is transition accuracy $v$ of the classification model and it is defined as

$$
v=\frac{e}{a}
$$


where $e$ is the number of estimated transitions, and $a$ is the actual number of transitions between indoor and outdoor locations. This metric evaluates the movement behaviour of the user with regards to their moving profile from indoors to outdoors and vice versa. The transitions are considered a net number. Finally, our analysis initially considers the complete dataset, and then we iteratively apply increasing subsampling to determine its effect on accuracy.

\section{RESULTS}

A total of 11 participants (6 male) were recruited (average age 27). In total, we collected 388 hours of data (97,498 data points), spanning a physical area of $81 \mathrm{sq}$. $\mathrm{km}$. We recorded 214 labels from participants (112 indoor). Those labels were indicated as 156 "now", 36 " 1 minute ago", 22 "5 minutes ago." During debriefing participants confirmed using their phones in a naturalistic manner, for example to make calls both indoors and outdoors, both with and without a headset. In addition, some biked outdoors, hiked, and took the bus during the experiment.

\subsection{Model 1: Indoor/outdoor classification}

For our classification and analysis, we used the Weka Machine Learning Toolbox in R. We experimented with a variety of available models, and J48 which is a Weka implementation of C4.5 proved to outperform other models. Hence, we report this classifier as our experimental classification model. Model 1 uses all available features, and is validated with a 10 -fold cross validation test. Our treated dataset had a frequency of $1 \mathrm{~Hz}$. We then performed subsampling on our data using steps of 2 to 100 . For example, subsampling of 10 means that we only retain 1 record every 10 seconds. In Fig. 3 (top-left), we show the prediction accuracy $(p)$ and confidence interval of our classifier model. When not performing subsampling, the prediction accuracy is $99 \%$, meaning that at any given moment the system is able to infer its surroundings with such accuracy. When subsampling, this value can drop to less than $40 \%$. In Fig. 3 (top-right) we present the results for transition accuracy $(v)$. When not performing subsampling, the transition accuracy is $100 \%$, meaning that instances of transitions to an indoor or outdoor setting are all correctly identified. When subsampling, this value can drop to $0 \%$.

\subsection{Model 2: feature selection}

Model 1 performs very well, but uses all features as input, and hence is not optimal in terms of energy consumption. Therefore, we performed feature selection to identify a smaller set of features which require fewer hardware sensors, and potentially reduce power consumption on smartphones. We experimented with multiple Weka feature selection algorithms and we achieved the best results with the Consistency Subset Evaluation model enhanced by Genetic Search.

After applying this feature selection model, the predictive attributes are reduced to: (1) Activity, (2) Barometric Pressure, (3) Ambient Luminance, (4) Accelerometer, (5) Magnetometer Variance and (6) Number of WiFi Access Points. Subsequently, we input these predictive attributes to a J48 classifier and we observed that the overall accuracy is comparable to that of Model 1.

We validate this model with a 10 -fold cross validation test. Again, we performed subsampling as reported earlier. In Fig. 3 (middle), we show the prediction accuracy $(p)$ which is $98.44 \%$ when not performing subsampling. When subsampling, this value can drop to less than $40 \%$. We also show the results for transition accuracy $(v)$. When not performing subsampling, the transition accuracy is $100 \%$, meaning that instances of transitions to an indoor or outdoor setting are identified correctly. When subsampling, this value can drop to $0 \%$.

\subsection{Model 3: energy efficient attributes}

The features used in Model 2 are chosen to maximise prediction accuracy. However, this does not explicitly consider the energy consumption of those features. To optimise power consumption, we further experimented by removing energy-intensive attributes from Model 2, to produce a heuristic model which is based on both high prediction accuracy and low power consumption.

This leads to Model 3, which uses the following predictive attributes: (1) Activity, (2) Ambient Luminance, and (3) Number of WiFi Access Points. Subsequently, we input these predictive attributes to a J48 classifier and we observed that the overall accuracy is comparable to Models 1 and 2. We validate this model with a 10 -fold cross validation test. Again, we performed subsampling as reported earlier. 
In Fig. 3 (bottom) we show the prediction accuracy $(p)$ which is $92.91 \%$ when not performing subsampling, and can drop to less than $60 \%$ when subsampling. The results for transition accuracy $(v)$ show that it is $100 \%$ without subsampling, and can drop to $0 \%$ when subsampling.

\subsection{Energy Consumption versus Prediction Accuracy}

Our work explicitly attempts to balance the trade-off between energy consumption and prediction accuracy for quantifying environmental exposure. While some previous work has noted that that energy efficiency can be increased by relying on lightweight smartphone sensors and subsampling [51], it is not clear what is the trade-off with the accuracy of the system. We have quantified how reducing the duty cycle of sensors can impact the accuracy of the system (Fig. 4) and reduce energy (Fig. 5).

\section{Prediction accuracy (p)}
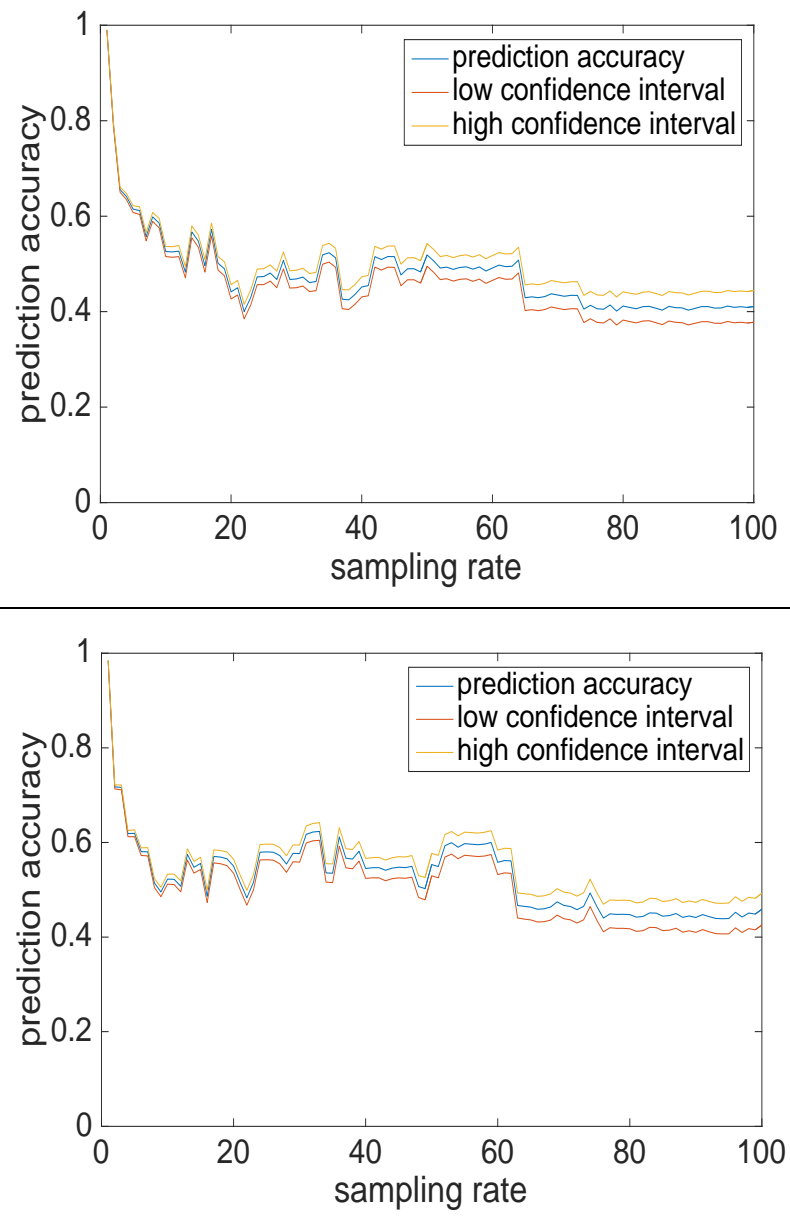

Transition accuracy (v)
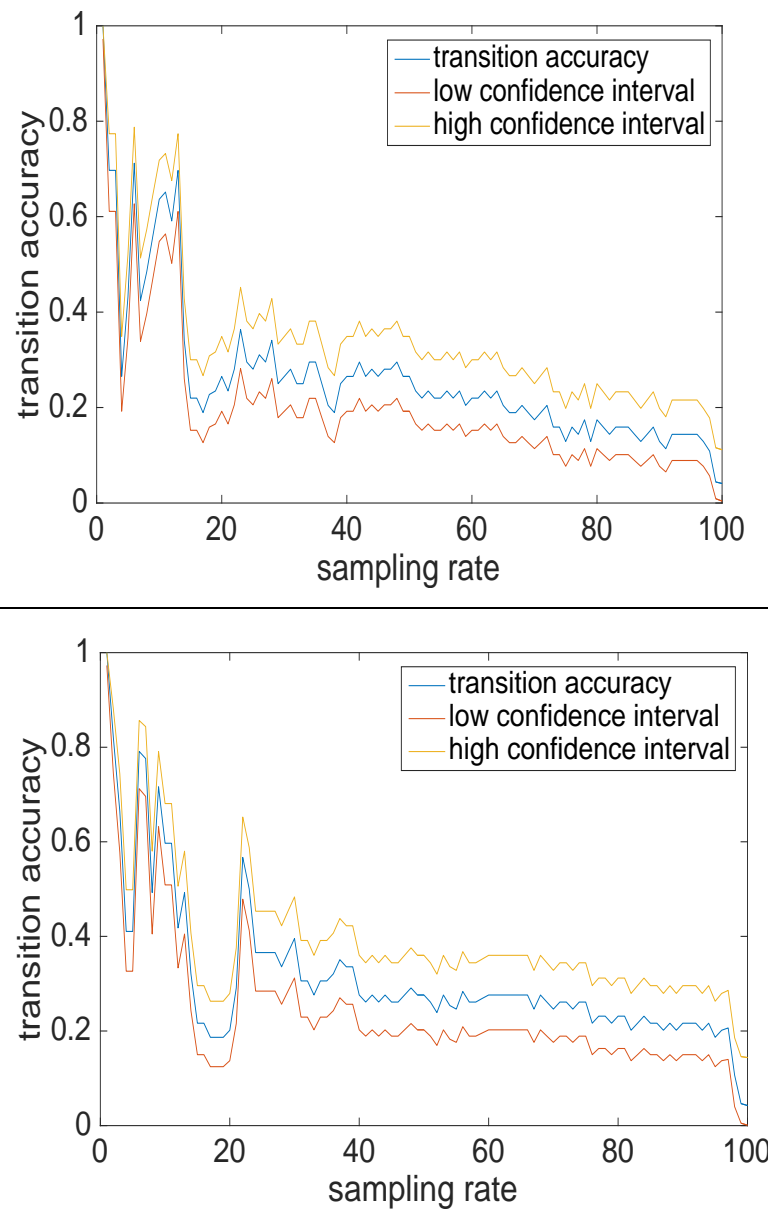

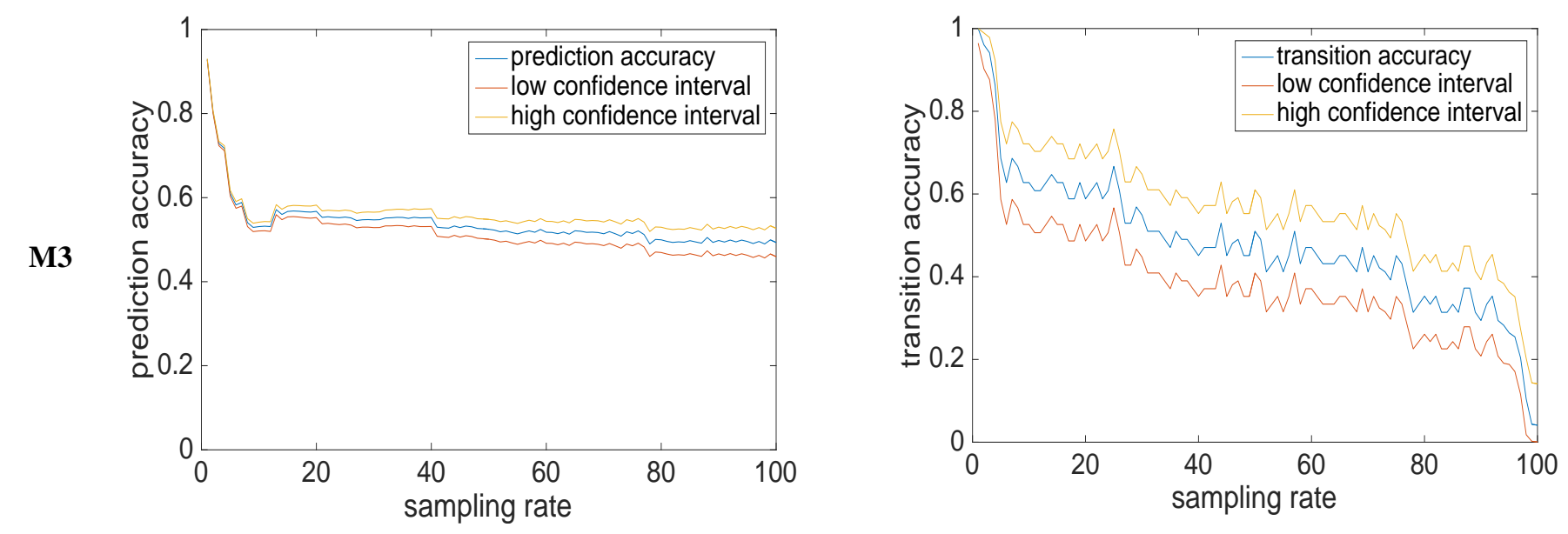

Fig. 3. Prediction accuracy of Models 1 (top), 2 (middle), and 3 (bottom).

The associated $95 \%$ confidence intervals are also shown. Sampling rates in Hz.

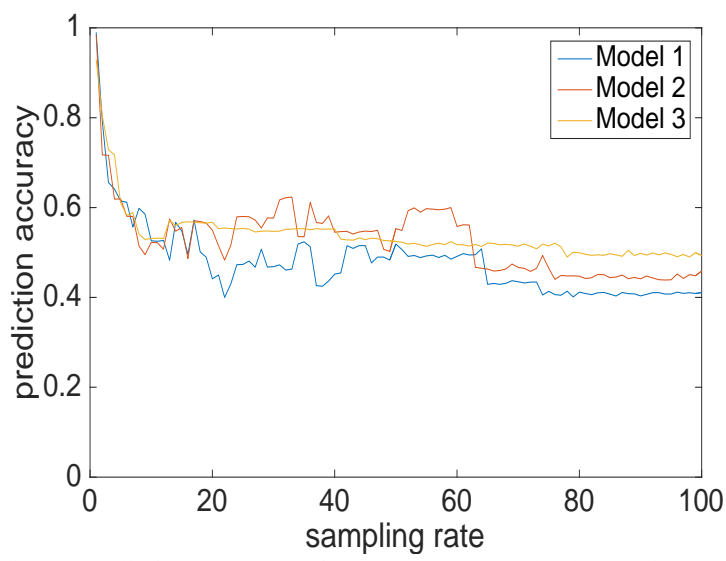

Fig. 4. Prediction accuracy of Models 1, 2, 3 and subsampling rate 1$100(\mathrm{~Hz})$.

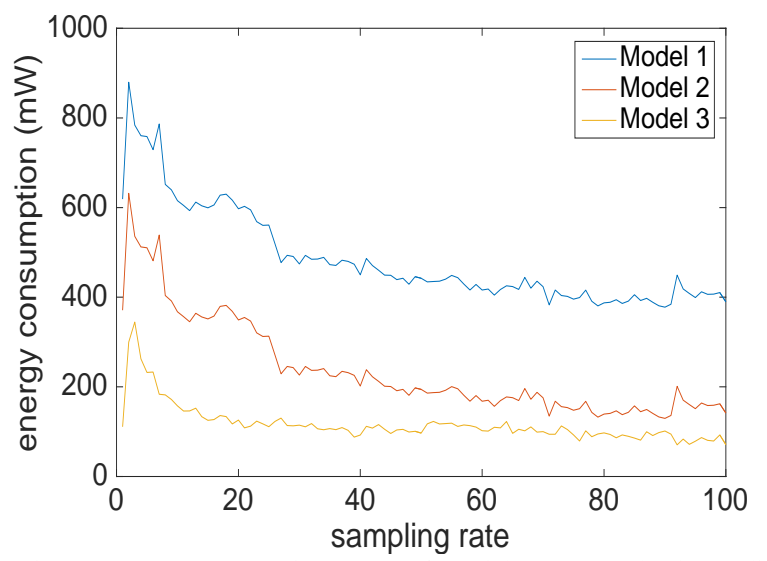

Fig. 5. Energy consumption (mW) of Models 1, 2, 3 and subsampling rate $1-100$.

To estimate the energy consumption (Fig. 5), Qualcomm's Trepn Power Profiler [42,43] is used, which provides the battery power consumption in $\mathrm{mW}$. The profiler was set to collect data every $100 \mathrm{~ms}$, following the software best practices [42]. To cycle through all our subsampling rates from 1 to 100, our software turned on and off the sensors according to the desired sampling rate for a period of 5 minutes. After this period, the subsampling rate was incremented by 1 , and the procedure was repeated accordingly.

- Model 1: we profiled the power consumption of simultaneously sensing barometric pressure, ambient light, acceleration and magnetic variance. The magnetometer is only turned on and off for subsampling rates greater than 18, since we require an 18-second window of magnetic variance. Because the remaining sensors of Model 1 are only recorded every 5 minutes in our deployment, we added to our power measurements the respective values from Table I.

- Model 2: we profiled the power consumption of simultaneously sensing barometric pressure, ambient light, acceleration and magnetic variance. The magnetometer is only turned on and off for subsampling rates greater than 18, since we require an 18-second window of magnetic variance. Because the remaining sensors of Model 2 are only recorded every 5 minutes in our deployment, we added to our power measurements the respective values from Table I. 
- Model 3: measurements included only the ambient luminance sensor. Because the remaining sensors of Model 3 are only recorded every 5 minutes in our deployment, we added to our power measurements the respective values from Table I.

\subsection{Extreme cases results}

We tested Model 3, which is our proposed model for quantifying environmental exposure, against the extreme cases dataset we recorded independently of our participants.

This dataset was not used in training any of our models. The extreme cases consisted of 6 scenarios (basement, near window, urban canyon, rapid transitions in/out, different country). In most scenarios, there were 2 conditions: having the phone in the pocket or holding it in hand. Model 3 was evaluated against these scenarios without training and with training (using a 10-fold cross validation test), as shown in Table II.

Table II

Summary of Model 3 prediction accuracy for the extreme cases dataset

\begin{tabular}{lccc} 
Setting & $\begin{array}{c}\text { Position of } \\
\text { phone }\end{array}$ & $\begin{array}{c}\text { Prediction accuracy } \\
\text { without training } \\
\mathbf{( \% )}\end{array}$ & $\begin{array}{c}\text { Prediction accuracy } \\
\text { with training (\%) }\end{array}$ \\
\multirow{2}{*}{ Basement } & Pocket & 24.86 & 100 \\
& Hand & 43.55 & 93.33 \\
\hline Near big & Pocket & 34.96 & 100 \\
windows & Hand & 14.09 & 91.23 \\
\hline Urban & Pocket & 81.68 & 98.99 \\
canyon & Hand & 100 & 100 \\
\hline In \& Out & Pocket & 52.41 & 62.89 \\
\hline Different & Hand & 47.92 & 98.35 \\
country & Mixed & 42.22 & \\
\hline
\end{tabular}

\subsection{Comparison with other models}

The performance of our models is shown in Table III, along with the performance of models reported in previous work. The energy consumption estimations are calculated by considering which sensors are used in each approach and their respective power needs (in Table I), and assuming no subsampling takes place. Our work is directly comparable to a handful of previous work that explicitly quantifies environmental exposure. Indicatively, we estimate that a patient's exposure is misclassified for 1 day per each person-year of observation with Models 1 and 2, and 14 days with Model 3.

Table III

Comparative assessment of environmental exposure methods

\begin{tabular}{ccccc} 
Approach & $\begin{array}{c}\text { Accuracy } \\
(\boldsymbol{\%})\end{array}$ & $\begin{array}{c}\text { Error } \\
\text { (days/year) }\end{array}$ & Energy $(\mathbf{m W})$ & Sensors used \\
Model 1 & 99 & 0.8 & 680.76 & All in Table I \\
\hline Model 2 & 98.44 & 1.0 & 370.96 & $\begin{array}{c}\text { Activity } \\
\text { Barometer } \\
\end{array}$ \\
& & & $\begin{array}{c}\text { Ambient luminance } \\
\text { Accelerometer } \\
\text { Magnetometer }\end{array}$ \\
\hline
\end{tabular}




\begin{tabular}{ccccc}
\hline & & & & WiFi \\
\hline Model 3 & 92.91 & 14.2 & 110.68 & $\begin{array}{c}\text { Activity } \\
\text { Ambient luminance } \\
\text { WiFi }\end{array}$ \\
\hline IODetector [21] & 85 & 54.6 & 218.62 & $\begin{array}{c}\text { Acceleration } \\
\text { Proximity } \\
\text { Ambient Light } \\
\text { GSM signal strength } \\
\text { Magnetometer }\end{array}$ \\
\hline Okamoto \& Chen [32] $86.1-96.5$ & 12.8 & 136.67 & $\begin{array}{c}\text { GPS } \\
\text { Magnetometer }\end{array}$ \\
\hline Xu et al. [49] & 90 & 36.5 & $>211$ & $\begin{array}{c}\text { GPS } \\
\text { Camera } \\
\text { Ambient luminance } \\
\text { WiFi } \\
\text { GSM signal strength } \\
\text { Magnetometer }\end{array}$ \\
\hline
\end{tabular}

For completeness, in Table IV and V, we include additional activity recognition and localisation techniques, even though they are not directly comparable. These can be used to extrapolate environmental exposure assuming that a reliable mapping exists between location (or activity) and environmental exposure.

Table IV

Comparative assessment of activity recognition methods

\begin{tabular}{ccc}
$\begin{array}{c}\text { Activity recognition } \\
\text { methods }\end{array}$ & Accuracy (\%) & $\begin{array}{c}\text { Error (days per person- } \\
\text { year of observation) }\end{array}$ \\
\hline$[27]$ & 84 & 58.4 \\
{$[38]$} & 92.33 & 28 \\
{$[34]$} & 90 & 36.5 \\
{$[23]$} & 90 & 36.5 \\
\hline
\end{tabular}

Table V

Comparative assessment of localization methods

\begin{tabular}{ccc}
$\begin{array}{c}\text { Localisation } \\
\text { methods }\end{array}$ & $\begin{array}{c}\text { Accuracy } \\
(\%)\end{array}$ & $\begin{array}{c}\text { Error (days per person- } \\
\text { year of observation) }\end{array}$ \\
{$[47]$} & 75 & 91.2 \\
{$[32]$} & 87.2 & 46.7 \\
{$[5]$} & 87 & 47.5 \\
{$[20]$} & 89 & 40.2 \\
{$[52]$} & 80 & 73 \\
{$[24]$} & 75 & 91.3 \\
{$[12]$} & $95.5-98$ & $7.3-16.4$ \\
{$[14]$} & 84 & 58.4 \\
{$[41]$} & 94.87 & 18.7 \\
{$[15]$} & $80-90$ & $36.5-73$ \\
{$[51]$} & 90.82 & 33.5 \\
{$[25]$} & 95 & 18.3 \\
\hline
\end{tabular}

\section{DISCUSSION}

Our work seeks to quantify environmental exposure using smartphones, and we consider both accuracy and energy efficiency as relevant criteria in our assessment. We have presented three models which progressively consume less energy, and we evaluate them against a range of subsampling strategies. Overall, we measure the accuracy of our 
models by considering their prediction accuracy $(p)$. Prediction accuracy is a measure often reported in prior work, and thus makes comparison to previous work possible. However, for health sciences it is also appropriate to provide a measure of confidence intervals, and we estimate those using a binomial experiment test. In addition, we provide a measure of transition accuracy $(v)$, which is relevant when quantifying the number of times that in individual was indoors or outdoors. Such a measure is relevant in behavioural studies and studies that are interested in detecting instances of exposure.

\subsection{Towards smartphone-based environmental exposure assessment}

Feasible and accurate environmental exposure detection has been an important challenge within the scientific community, as denoted by the large quantity of work in this area $[21,28,32,34,49]$. However, a suitable and reliable method has been elusive. The majority of current approaches rely on unrealistic set of conditions that are normally too cumbersome and do not scale well. For instance, it quickly becomes unfeasible beyond small controlled probes to require instrumentation of the environment through the installation of beacons/tags or an a priori mapping of the environment are required.

Since smartphones are carried by users daily [13] and are fitted with a several different sensors, smartphones are ideal for feasible and accurate environmental exposure detection. Due to their ubiquity, a practical method that leverages smartphones can provide crucial methodological advances to scientists who seek to quantify environmental exposure through an affordable, easy to automate and longitudinal monitoring technique. Other examples of stakeholders that could benefit greatly from smartphone Indoor/Outdoor detection are cellular service providers and those studying human mobility patterns [21].

In addition, users can benefit from collecting indoor/outdoor data on their smartphones since they can enhance their spatial context towards more location-based services, such as better reminders, and personal assistance applications. However, for the model to be valuable to users, it is likely to require some initial training since activity recognition is well aligned with the behavioural patterns of certain human entities. This is the reason why Model 3 does not scale well with data from a different spatial context, like the extreme cases and the different country datasets. To overcome these weaknesses, one must train the model according to each user's behaviour, which tends to be routine and likely predictable. A smartphone can use notifications to collect ground-truth during training of the system, perhaps as a go-to action when the phone is unlocked [44].

\subsection{Energy-efficient inference on smartphones}

While smartphones have several advantages in an Indoor/Outdoor detection scenario, it is important to consider the challenge of energy-efficiency. For instance, previous work has tried to improve activity recognition models to reduce energy consumption while still maintaining acceptable prediction accuracy $[8,38]$. Here, we approach indoor/outdoor detection of a mobile user as a classification problem and explicitly attempt to balance the trade-off between power consumption and prediction accuracy for quantifying environmental exposure.

Our work seeks to quantify environmental exposure using smartphones, and we consider both prediction accuracy and energy efficiency as relevant criteria in our assessment. We have quantified the relationship between prediction accuracy and power consumption with off-the-shelf smartphones without the need to use specific lightweight smartphone sensors as has been proposed in previous work [51].

At the same time, reliable indoor/outdoor information can also be highly useful for the energy-efficiency of other devices. For example, cameras whose energy consumption and processing time depend on the ambient environment can achieve better efficiency and performance with primitive indoor/outdoor information [21].

When considering a traditional machine learning approach, like the case of Models 1 and 2, the main interest is in defining, via feature selection, the best subset of features which produce the highest prediction accuracy. However, little research has considered energy efficiency in the context of optimising machine learning models. In order to achieve lower energy consumption and high prediction accuracy, we extended the techniques of machine learning approaches by introducing a heuristic that was based on the observation of the energy consumption of certain smartphone sensors. This lead to the formulation of Model 3, which traditional machine learning approaches could not infer for our domain-specific problem. The heuristics of this approach are based on the fact that we treat machine learning features as smartphone sensors which can be dynamically switched on/off to enrich the sensed context. We feel that the energy optimisation of classifiers on mobile handsets is a promising direction for further systematic investigation. 


\subsection{Limitations}

The proposed energy efficient model is stateful, meaning that it is trained on a certain dataset and does not adapt to new unseen indoor/outdoor patterns instantly. This limitation explains the low prediction accuracy observed with the extreme cases. Specifically, if the model was stateless and was incrementally adaptive to new unseen indoor/outdoor patterns, its behaviour at the extreme cases crash test would be improved over time. In addition, the model needs maintenance, by periodically incorporating new unseen indoor/outdoor patterns, which is computationally inefficient given the resource restrictions on smartphones. Both these limitations can be addressed by server offloading.

\section{CONCLUSION}

In this paper, we approach indoor/outdoor detection of a mobile user as a binary classification problem. To classify the environmental context of the user, we build a model which accepts as input multiple contextual features and outputs a class attribute, which is the Indoor/Outdoor feature. We build three Models of varying prediction accuracy and energy consumption on smartphones. We evaluate their prediction accuracy, transition accuracy and power efficiency by using real traces and also by applying subsampling. We identified a relation between prediction accuracy and power consumption, and using this trade-off we evaluated our models in relation to other models reported the literature. Our work balances the trade-off between power consumption and accuracy for quantifying environmental exposure. Ultimately a power-efficient and accurate model can be used in a range of human sciences studies, and provide significant methodological advances to the study of environmental exposure.

\section{ACKNOWLEDGEMENTS}

This work is partially funded by the Academy of Finland (Grants 276786-AWARE, 286386-CPDSS, 285459iSCIENCE, 304925-CARE), the European Commission (Grant 6AIKA-A71143-AKAI), and Marie SkłodowskaCurie Actions (645706-GRAGE)

\section{REFERENCES}

[1] Almanac for Computers, 1990. Nautical Almanac Office, US Naval Observatory, Washington, D.C.

[2] ActivityRecognitionApi | Google $\quad$ APIs for https://developers.google.com/android/reference/com/google/android/gms/location/ActivityRecognitionApi\#public-methods, retrieved 21/05/2016.

[3] John L. Adgate, Timothy R. Church, Andrew D. Ryan, Gurumurthy Ramachandran, et al. 2004. Outdoor, indoor, and personal exposure to VOCs in children. Environmental Health Perspectives, 1386-1392.

[4] Denzil Ferreira, Vassilis Kostakos and Anind K. Dey. 2015. AWARE: Mobile Context Instrumentation Framework. Frontiers in ICT, 2 (6). 1-9. DOI: https://doi.org/10.3389/fict.2015.00006

[5] Martin Azizyan, Ionut Constandache and Romit Roy Choudhury. 2009. SurroundSense: Mobile Phone Localization via Ambience Fingerprinting. In Proceedings of the 15th International Conference on Mobile Computing and Networking, ACM, 261-272. http://dx.doi.org/10.1145/1614320.1614350.

[6] Peter A. Baghurst, Anthony J. McMichael, Neil R. Wigg, Graham V. Vimpani, Evelyn F. Robertson, Russell J. Roberts and Shi-Lu Tong. 1992. Environmental Exposure to Lead and Children's Intelligence at the Age of Seven Years. N Engl J Med 327, 18, 1279-1284. http://dx.doi.org/10.1056/nejm199210293271805.

[7] Joel Barnes, Chris Rizos, Jinling Wang, David Small, Gavin Voigt and Nunzio Gambale. 2002. High precision indoor and outdoor positioning using LocataNet. Positioning 1, 05.

[8] Fehmi Ben Abdesslem, Andrew Phillips and Tristan Henderson. 2009. Less is More: Energy-efficient Mobile Sensing with Senseless. In Proceedings of the 1st ACM Workshop on Networking, Systems, and Applications for Mobile Handhelds, 6162. http://dx.doi.org/10.1145/1592606.1592621.

[9] Gennady Berkovich. 2014. Accurate and reliable real-time indoor positioning on commercial smartphones. In International Conference on Indoor Positioning and Indoor Navigation, IEEE, 670-677. http://dx.doi.org/10.1109/IPIN.2014.7275542.

[10] Ralf Bill, Clemens Cap, Martin Kofahl and Thomas Mundt. 2004. Indoor and outdoor positioning in mobile environmentsa review and some investigations on wlan-positioning. Geographic Information Sciences 10, 2. 
[11] Ke-Yu Chen, Mark Harniss, Justin Lim, Youngjun Han, Kurt Johnson and Shwetak Patel. 2013. uLocate: A Ubiquitous Location Tracking System for People Aging with Disabilities. In Proceedings of the International Conference on Body Area Networks, 173-176. http://dx.doi.org/10.4108/icst.bodynets.2013.253584.

[12] Sung-Bae Cho. 2015. Exploiting machine learning techniques for location recognition and prediction with smartphone logs. Neurocomputing 176, C, 98-106. http://dx.doi.org/10.1016/j.neucom.2015.02.079.

[13] Anind K. Dey, Katarzyna Wac, Denzil Ferreira, Kevin Tassini, Jin-Hyuk Hong and Julian Ramos. 2011. Getting closer: an empirical investigation of the proximity of user to their smart phones. In International Conference on Ubiquitous Computing, ACM, 163-172. http://dx.doi.org/10.1145/2030112.2030135.

[14] Trinh Do, Olivier Dousse, Markus Miettinen and Daniel Gatica-Perez. 2015. A probabilistic kernel method for human mobility prediction with smartphones. Pervasive and Mobile Computing 20, 13-28. http://dx.doi.org/10.1016/j.pmcj.2014.09.001.

[15] Md O. Gani, Casey O'Brien, S. I. Ahamed and Roger O. Smith. 2013. RSSI Based Indoor Localization for Smartphone Using Fixed and Mobile Wireless Node. In Computer Software and Applications Conference, IEEE, 110-117. http://dx.doi.org/10.1109/COMPSAC.2013.18.

[16] M. I. Gilmour, Maritta S. Jaakkola, Stephanie J. London, Andre A. E. Nel and Christine C. A. Rogers. 2006. How exposure to environmental tobacco smoke, outdoor air pollutants, and increased pollen burdens influences the incidence of asthma. Environmental Health Perspectives, 627-633.

[17] Jorge Goncalves, Zhanna Sarsenbayeva, Niels van Berkel, Chu Luo, Simo Hosio, Sirkka Risanen, Hannu Rintamäki and Vassilis Kostakos. 2017. Tapping Task Performance on Smartphones in Cold Temperature. Interacting with Computers, 29(3), 355-367.

[18] Kembra L. Howdeshell, Andrew K. Hotchkiss, Kristina A. Thayer, John G. Vandenbergh and Frederick S. Vom Saal. 1999. Environmental toxins: exposure to bisphenol A advances puberty. Nature 401, 6755, 763-764.

[19] Simon Klakegg, Jorge Goncalves, Niels van Berkel, Chu Luo, Simo Hosio and Vassilis Kostakos. 2017. Towards Commoditised Near Infrared Spectroscopy. In Proceedings of the ACM SIGCHI Conference on Designing Interactive Systems, to appear.

[20] Jenq-Shiou Leu, Min-Chieh Yu and Hung-Jie Tzeng. 2015. Improving indoor positioning precision by using received signal strength fingerprint and footprint based on weighted ambient Wi-Fi signals. Computer Networks 91, 329-340. http://dx.doi.org/10.1016/j.comnet.2015.08.032.

[21] Mo Li, Pengfei Zhou, Yuanqing Zheng, Zhenjiang Li and Guobin Shen. 2014. IODetector: A Generic Service for Indoor/Outdoor Detection. ACM Trans. Sen. Netw 11, 2, 28:1-28:29. http://dx.doi.org/10.1145/2659466.

[22] Lighting Standard.
http://www.etaplighting.com/uploadedFiles/Downloadable_documentation/documentatie/EN12464_E_OK.pdf

[23] A. Lindo, Maria del Carmen Perez, J. Urena, David Gualda, Eloy Garcia and J. M. Villadangos. 2014. Ultrasonic signal acquisition module for smartphone indoor positioning. Emerging Technology and Factor Automation, 1-4.

[24] Guangwen Liu, Masayuki Iwai, Yoshito Tobe, Dunstan Matekenya, Khan Hossain, Masaki Ito and Kaoru Sezaki. 2014. Beyond Horizontal Location Context: Measuring Elevation Using Smartphone's Barometer. In Proceedings of the 2014 ACM International Joint Conference on Pervasive and Ubiquitous Computing: Adjunct Publication, ACM, $459-468$. http://dx.doi.org/10.1145/2638728.2641670.

[25] Sérgio I. Lopes, José M. Vieira, João Reis, Daniel Albuquerque and Nuno B. Carvalho. 2015. Accurate smartphone indoor positioning using a WSN infrastructure and non-invasive audio for TDoA estimation. Pervasive and Mobile Computing 20, 29-46. http://dx.doi.org/10.1016/j.pmcj.2014.09.003.

[26] Weiwei Jiang, Denzil Ferreira, Jani Ylioja, Jorge Goncalves and Vassilis Kostakos. 2014. Pulse: Low Bitrate Wireless Magnetic Communication for Smartphones. In Proceedings of the 2014 ACM International Joint Conference on Pervasive and Ubiquitous Computing, ACM, 261-265.

[27] Hong Lu, Jun Yang, Zhigang Liu, Nicholas D. Lane, Tanzeem Choudhury and Andrew T. Campbell. 2010. The Jigsaw Continuous Sensing Engine for Mobile Phone Applications. In Proceedings of the 8th ACM Conference on Embedded Networked Sensor Systems, ACM, 71-84. http://dx.doi.org/10.1145/1869983.1869992.

[28] Hiroshi Mizuno, Ken Sasaki and Hiroshi Hosaka. 2007. Indoor-outdoor Positioning and Lifelog Experiment with Mobile Phones. In Proceedings of the 2007 Workshop on Multimodal Interfaces in Semantic Interaction, ACM, 55-57. http://dx.doi.org/10.1145/1330572.1330582.

[29] Christian Monn. 2001. Exposure assessment of air pollutants: a review on spatial heterogeneity and indoor/outdoor/personal exposure to suspended particulate matter, nitrogen dioxide and ozone. Atmospheric Environment 35, 1, 1-32. http://dx.doi.org/10.1016/s1352-2310(00)00330-7.

[30] Pavan K. Namineni, Trevor Davey, Gary Siebert and Charles J. Jacobus. 2010. Wireless mobile indoor/outdoor tracking system. Patent US 7852262 B2

[31] Lionel Ni, Yunhao Liu, Yiu C. Lau and Abhishek A. P. Patil. 2004. LANDMARC: indoor location sensing using active RFID. Wireless Networks 10, 6, 701-710.

[32] Masayuki Okamoto and Cheng Chen. 2015. Improving GPS-based Indoor-outdoor Detection with Moving Direction Information from Smartphone. In Adjunct Proceedings of the 2015 ACM International Joint Conference on Pervasive and Ubiquitous Computing, ACM, 257-260. http://dx.doi.org/10.1145/2800835.2800939. 
[33] Eamonn O'Neill, Vassilis Kostakos, T. Kindberg, A. Schiek, A. Penn, D. Fraser and T. Jones. 2006. Instrumenting the city: Developing methods for observing and understanding the digital cityscape. In International Conference on Ubiquitous Computing, Springer, 315-332. http://dx.doi.org/10.1007/11853565_19.

[34] Kazushige Ouchi and Miwako Doi. 2012. Indoor-outdoor Activity Recognition by a Smartphone. In Proceedings of the 2012 ACM Conference on Ubiquitous Computing, ACM, 537-537. http://dx.doi.org/10.1145/2370216.2370297.

[35] Svati Patandin, Corine Koopman-Esseboom, Maria De Ridder, Nynke Weisglas-Kuperus and Pieter Sauer. 1998. Effects of Environmental Exposure to Polychlorinated Biphenyls and Dioxins on Birth Size and Growth in Dutch Children. Pediatr Res 44, 4, 538-545. http://dx.doi.org/10.1203/00006450-199810000-00012.

[36] Svati Patandin, Caren I. Lanting, Paul G. Mulder, E. Rudy Boersma, Pieter J. Sauer and Nynke Weisglas-Kuperus. 1999. Effects of environmental exposure to polychlorinated biphenyls and dioxins on cognitive abilities in Dutch children at 42 months of age. The Journal of Pediatrics 134, 1, 33-41.

[37] Zhanna Sarsenbayeva, Jorge Goncalves, Juan García, Simon Klakegg, Sirkka Rissanen, Hannu Rintamäki, Jari Hannu and Vassilis Kostakos. 2016. Situational Impairments to Mobile Interaction in Cold Environments. In Proceedings of the 2016 ACM International Joint Conference on Pervasive and Ubiquitous Computing, ACM, 85-96.

[38] Valentin Radu, Panagiota Katsikouli, Rik Sarkar and Mahesh K. Marina. 2014. A Semi-supervised Learning Approach for Robust Indoor-outdoor Detection with Smartphones. In Proceedings of the 12th ACM Conference on Embedded Network Sensor Systems, ACM, 280-294.

[39] Paul Schlyter. 2010. Radiometry and photometry in astronomy, retrieved 12/08/2016.

[40] Sunrise/Sunset Algorithm. http://williams.best.vwh.net/sunrise_sunset_algorithm.htm, retrieved 18/08/2016.

[41] Joaquín Torres-Sospedra, Raúl Montoliu, Sergio Trilles, Óscar Belmonte and Joaquín Huerta. 2015. Comprehensive analysis of distance and similarity measures for Wi-Fi fingerprinting indoor positioning systems. Expert Systems with Applications 42, 23, 9263-9278. http://dx.doi.org/10.1016/j.eswa.2015.08.013.

[42] Trepn Power Profiler - Qualcomm Developer Network. https://developer.qualcomm.com/software/trepn-power-profiler, retrieved 27/05/2016.

[43] Trepn Profiler - Android. https://play.google.com/store/apps/details?id=com.quicinc.trepn, retrieved 27/05/2016.

[44] Khai N. Truong, Thariq Shihipar and Daniel J. Wigdor. 2014. Slide to X: unlocking the potential of smartphone unlocking. In Proceedings of the 32nd annual ACM conference on Human factors in computing systems, 3635-3644.

[45] Karel Van den Broucke, Denzil Ferreira, Jorge Goncalves, Vassilis Kostakos and Katrien De Moor. 2014. Mobile Cloud Storage: A Contextual Experience. In International Conference on Human-Computer Interaction with Mobile Devices and Services, ACM, 101-110. http://dx.doi.org/10.1145/2628363.2628386.

[46] Fan Wang, Zhengyong Huang, Hui Yu, Xiaohua Tian, Xinbing Wang and Jinwei Huang. 2013. EESM-based fingerprint algorithm for Wi-Fi indoor positioning system. In International Conference on Communications in China, IEEE, 674-679. http://dx.doi.org/10.1109/ICCChina.2013.6671197.

[47] Yi Wang, Jialiu Lin, Murali Annavaram, Quinn A. Jacobson, Jason Hong, Bhaskar Krishnamachari and Norman Sadeh. 2009. A framework of energy efficient mobile sensing for automatic user state recognition. In Proceedings of the 7 th international conference on Mobile systems, applications, and services, 179-192. http://dx.doi.org/10.1145/1555816.1555835.

[48] Wasiq Waqar, Yuanzhu Chen and Andrew Vardy. 2016. Smartphone positioning in sparse Wi-Fi environments. Computer Communications 73, 108-117. http://dx.doi.org/10.1016/j.comcom.2015.09.002.

[49] Wenchao Xu, Ruizhi Chen, Tianxing Chu, Lei Kuang, et al. 2014. A context detection approach using GPS module and emerging sensors in smartphone platform. In Ubiquitous Positioning Indoor Navigation and Location Based Service, IEEE, 156-163.

[50] Zheng Yang, Chenshu Wu and Yunhao Liu. 2012. Locating in Fingerprint Space: Wireless Indoor Localization with Little Human Intervention. In Proceedings of the 18th International Conference on Mobile Computing and Networking, ACM, 269-280. http://dx.doi.org/10.1145/2348543.2348578.

[51] Dezhong Yao, Chen Yu, Anind A. K. Dey, Christian Koehler, Geyong Min, Laurence L. T. Yang and Hai Jin. 2014. Energy efficient indoor tracking on smartphones. Future Generation Computer Systems 39, 44-54. http://dx.doi.org/10.1016/j.future.2013.12.032.

[52] Zengbin Zhang, Xia Zhou, Weile Zhang, Yuanyang Zhang, Gang Wang, Ben B. Y. Zhao and Haitao Zheng. 2011. I Am the Antenna: Accurate Outdoor AP Location Using Smartphones. In Proceedings of the 17th Annual International Conference on Mobile Computing and Networking, ACM, 109-120. http://dx.doi.org/10.1145/2030613.2030626. 